\title{
Vibration Control and Design of Vehicle Engine Mount Activated by Magnetorheological Fluid
}

\author{
Zhaoxue Deng ${ }^{1, a}$, Ling Zheng ${ }^{1, b}$ \\ ${ }^{1}$ State Key Laboratory of Mechanical Transmission, Chongqing University, Chongqing, 400044, \\ China \\ adengzhaoxue@126.com, bzling@cqu.edu.cn
}

Key words: Vehicle engine, magnetorheological mount, LQR, fuzzy-logic controller

\begin{abstract}
Magneto-rheological (MR) fluid mount demonstrates excellent performance of vibration isolation in a wide frequency range and potential application in engineering. In this paper, a new mixed mode MR mount is proposed and an analytical model is established and used to predict the performance of MR mount. Meantime, a six degrees of freedom dynamic model of an in-line four-cylinder engine which has three points mounting system is derived by considering the dynamic behaviors of MR mount and its state space form is established. LQR and fuzzy-logic controllers are designed and synthesized to actively control the imposed vibration. The simulation results show that the proposed LQR or fuzzy-logic controllers can isolate the vibration of vehicle engine in a wide frequency range. The amplitudes can be suppressed well by employing this mount system. Its effectiveness will be verified via experiment in the second part of this work.
\end{abstract}

\section{Introduction}

An engine is one of the most dominant noise and vibration sources in vehicle systems. Therefore, in order to resolve noise and vibration problems generated by vehicle engine, various types of engine mounts have been proposed. The passive rubber engine mounts which has low damping, shows efficient vibration isolation performance in the non-resonant and high frequency excitation. However, it does not have a favorable performance at the resonant frequency excitation $[1,2]$. On the other hand, the efficiency of passive hydraulic engine mount in the non-resonant domain is worse than rubber mount. In order to overcome the limited performance of conventional passive mounts, semi-active mounts utilizing magnetorheological (MR) fluids have been developed for the purpose of vibration attenuation of dynamic systems.

In this work, a new type of MR mount, which combines the flow and shear modes, is proposed to reduce the vibration of the engine. This paper establishes six degrees of freedom model of the engine MR mount system, the conventional LQR controller and fuzzy controller is then designed to control unwanted vibration response of the engine. The controllers are experimentally implemented and control performances such as displacement and velocity are evaluated and presented in frequency domain.

\section{Design of the MR mount}

Figure 1 shows the schematic configuration of the mixed mode MR mount. The MR fluid is filled in the gap between the piston and the outer cylindrical housing. The electromagnetic coil is wired inside of the piston. The housing can be fixed to the supporting structure, and the piston is attached to the top end of the rubber element [3]. This rubber element has a role to support the static mass and isolate the vibration transmission at the non-resonant frequency regions. During the relative motion of the inner piston and outer cylindrical housing, the MR fluid flows through the annular gap. If a certain level of magnetic field is applied through the gap, the MR mount produces an additional damping force due to the yield stress of the MR fluid. 


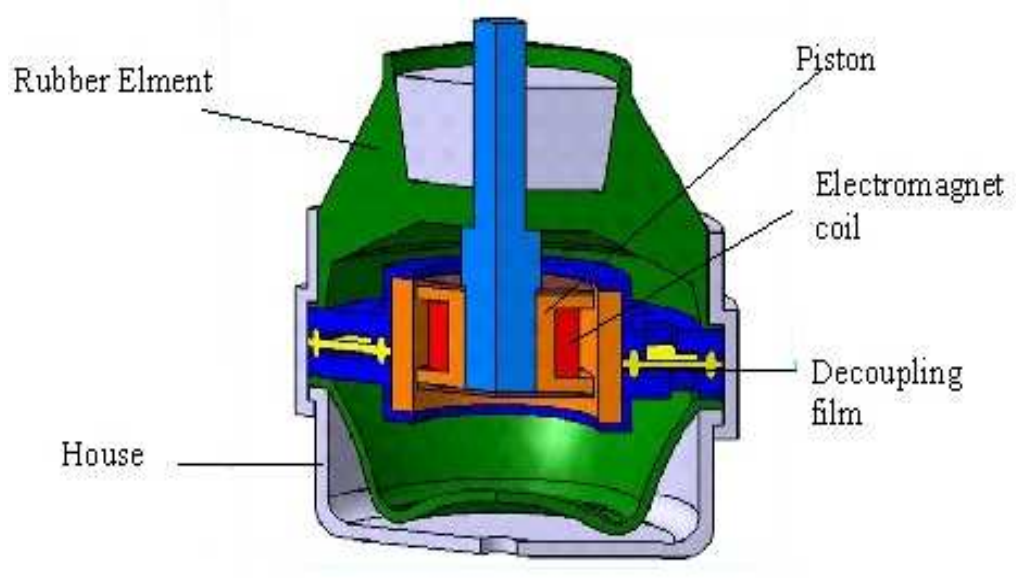

Fig. 1. Configuration of MR fluid mount

Magnetic field analysis is conducted to identify the magnetic flux density distribution of the damping channel, Simulation have been conducted using Ansys software. Material physical parameters use in magnetic field analysis according to tables 1 through 2, the cylindrical coil made of 150 turns wire. The simulation results show below have been generated for 1Amp passing through the coils.

Table 1 MRF

\begin{tabular}{lcccccccc}
\hline $\mathrm{H}(\mathrm{KA} / \mathrm{m})$ & 30 & 60 & 90 & 150 & 210 & 270 & 300 \\
\hline $\mathrm{B}(\mathrm{T})$ & 0.32 & 0.43 & 0.52 & 0.66 & 0.77 & 0.87 & 0.9 \\
\hline \multicolumn{7}{c}{ Table 2 Electrical pure iron (DT4) } \\
\hline $\mathrm{H}(\mathrm{KA} / \mathrm{m})$ & 21.2 & 42 & 84.8 & 104 & 304 & 1600 \\
\hline $\mathrm{B}(\mathrm{T})$ & 0.2 & 0.4 & 0.8 & 1 & 1.45 & 1.6 \\
\hline
\end{tabular}

Figures 2 shows simulated magnetic flux distributed through the MR fluid flowing inside the damping channel when the coil current is 1 Amp. Damping channel of magnetic flux density is 0.48 $\mathrm{T}$ closing to Magnetic saturation point of the MR fluid. The results show the simulated magnetic flux density increase with the generated current, so the MR mount can provides continuous damping force.

The control coefficient of the MR mount $(\mathrm{E})$ is represented as:

$$
E=\frac{F_{1}}{F_{2}+K_{r} x+C_{r} v} \approx \frac{F_{1}}{F_{2}+K_{r} x}
$$

In the above equation, $K_{r}$ is the stiffness of rubber, $C_{r}$ is the damping of rubber, $F_{1}$ is the viscous damping force, $F_{2}$ is the coulomb damping force.

Figure 3 shows accompanying with the increase of the current, the control coefficient of the MR mount increase. The MR mount has favorable adjustable in a wide frequency [4]. 

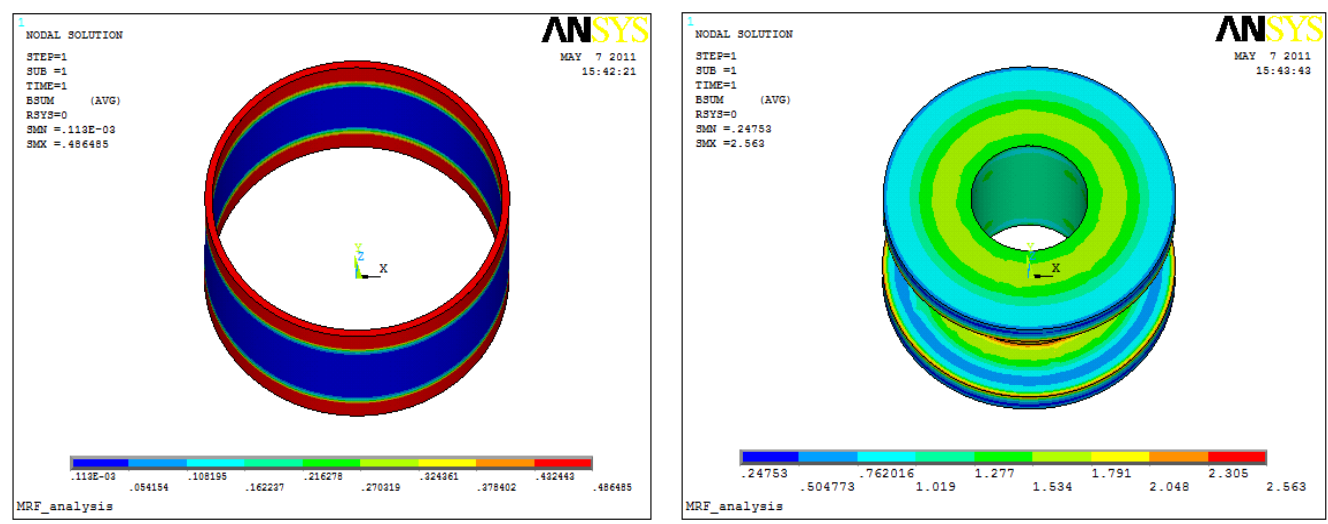

Fig. 2. Magnetic flux density distribution within damping channel and piston

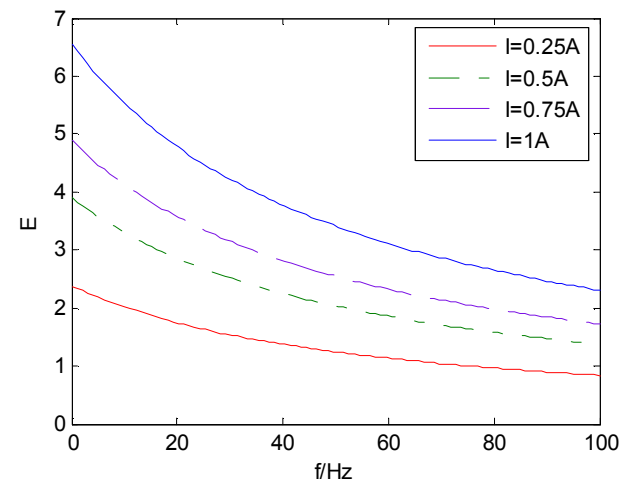

Fig. 3. The control coefficient of the Magnetorheological hydraulic mount

\section{Control strategy}

Engine model with MR mount. The six degrees-of-freedom model of an in-line four-cylinder engine is shown in Figure 4. The engine is supportted by three MR mounts. No.1 and No.2 MR engine mounts are situated at the bank and front left corner, respectively and No.3 MR engine mount is installed at the right edge of the engine in the $(\mathrm{x}, \mathrm{z})$ plane.

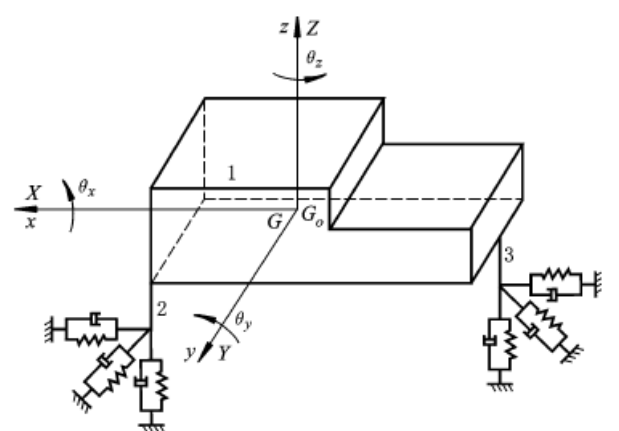

Fig. 4. The model of mounting system follow:

The six degrees-of-freedom dynamic equation is derived based on Lagrange's equation as

$$
\left[M_{s}\right]\{\ddot{p}\}+\left[C_{s}\right]\{\dot{p}\}+\left[K_{s}\right]\{p\}=\{F(t)\}+\left[B_{s}\right] U
$$

In order to solve the equations of the equation of the motion, some related parameters need to be determined. 


$$
\left[M_{s}\right]=\left[\begin{array}{ccccc}
m & & & & \\
& m & & & \\
& m & & & \\
& & J_{x} & -J_{x y} & -J_{z x} \\
& & -J_{x y} & J_{y} & -J_{y z} \\
& & -J_{z x} & -J_{y z} & J_{z}
\end{array}\right] \quad \mathrm{B}_{\mathrm{s}}=\left[\begin{array}{ccc}
0 & 0 & 0 \\
0 & 0 & 0 \\
-1 & -1 & -1 \\
-y 1 & -y 2 & -y 3 \\
x 1 & x 2 & x 3 \\
0 & 0 & 0
\end{array}\right] \quad\left[B_{i}\right]=\left[\begin{array}{cccccc}
1 & 0 & 0 & 0 & z i & -y i \\
0 & 1 & 0 & -z i & 0 & x i \\
0 & 0 & 1 & y i & -x i & 0
\end{array}\right]
$$

$$
\left[K_{s}\right]=\sum_{i=1}^{n}\left[B_{i}^{T}\right]\left[k_{i}\right]\left[B_{i}\right],[F(t)]^{T}=\left\{\begin{array}{lllllll}
0 & F_{y} & F_{z} & M_{x} & M_{y} & M_{Z}
\end{array}\right\}
$$

In the equation above, $M_{s}$ is the mass matrix, $C_{s}$ is the damping matrix, $K_{s}$ is the stiffness matrix, $F(t)$ is the external stimulation matrix, $\mathrm{B}_{\mathrm{s}}$ is the input matrix of controlling force.

To select the variables $X=\left[\begin{array}{ll}p & \dot{p}\end{array}\right]^{T}$, the status equation of mounting system is:

$$
\dot{X}=A X+B U+H F
$$

Considering the vibration signals at the centre of mass can't be measured directly, the vibration signal at the mount point is chosen to replace the vibration signal at the centre of mass. That means the vertical moving signal is regarded as the feedback signal of system. The output equation is:

$$
Y=C X
$$

Design of the optimal controller and fuzzy controller. The control magnetic field applied to the MR engine mount is determined by adopting the semi-active optimal controller [5-6]. The desired damping force is set by equation (5).

$$
u_{i}(t)=\left\{\begin{array}{ccc}
u_{i}(t) & \text { for } & u_{i} \cdot \dot{z}_{i}<0 \\
0 & \text { for } & u_{i} \cdot \dot{z}_{i}>0
\end{array} \quad(i=1,2,3)\right.
$$

where $u_{i}$ is the control force, $\dot{z}_{i}$ is the vertical velocity, the above condition physically implies that the control $u_{i}$ only assures the increment of energy dissipation of the stable system $[5,6]$. The weighted coefficients of optimal control are determined when the velocity of crank shaft is $650 \mathrm{r} / \mathrm{min}$.

The velocity range of crank shaft is $0-3000 \mathrm{r} / \mathrm{min}$. When designing fuzzy controller, the vertical velocity $v$ and the acceleration $a$ at mount point are selected as input of fuzzy controller, the output is the controllable damping force $u$ of MR mount. According to experience and theory, the fuzzy control rule is established. When the velocity and acceleration of MR mount point has the maximum negative value, the damping force value must reach maximum positive value.

Simulation experiment. Adopting Matlab simulation software, the simulation model of passive hydraulic mounting system and MR hydraulic mounting system is established. In the simulation period, the vibration signals of vertical displacement and velocity at a certain MR mount point is measured and presented in Figure 5 and 6 when the velocity range of crank shaft is $650 \mathrm{r} / \mathrm{min}$ and $1500 \mathrm{r} / \mathrm{min}$ respectively.

From the power spectrum of a certain MR mount point under different crank shaft rotating velocity, it can be clearly observed that the vibration velocity amplitude of passive hydraulic mount is respectively $0.038 \mathrm{~m} / \mathrm{s}$ and $0.023 \mathrm{~m} / \mathrm{s}$; the optimal control MR mount respectively $0.028 \mathrm{~m} / \mathrm{s}$ and $0.02 \mathrm{~m} / \mathrm{s}$; the fuzzy control MR mount respectively $0.024 \mathrm{~m} / \mathrm{s}$ and $0.018 \mathrm{~m} / \mathrm{s}$. Compared the above results, the design of optimal control and fuzzy control rule is rational and effective. 

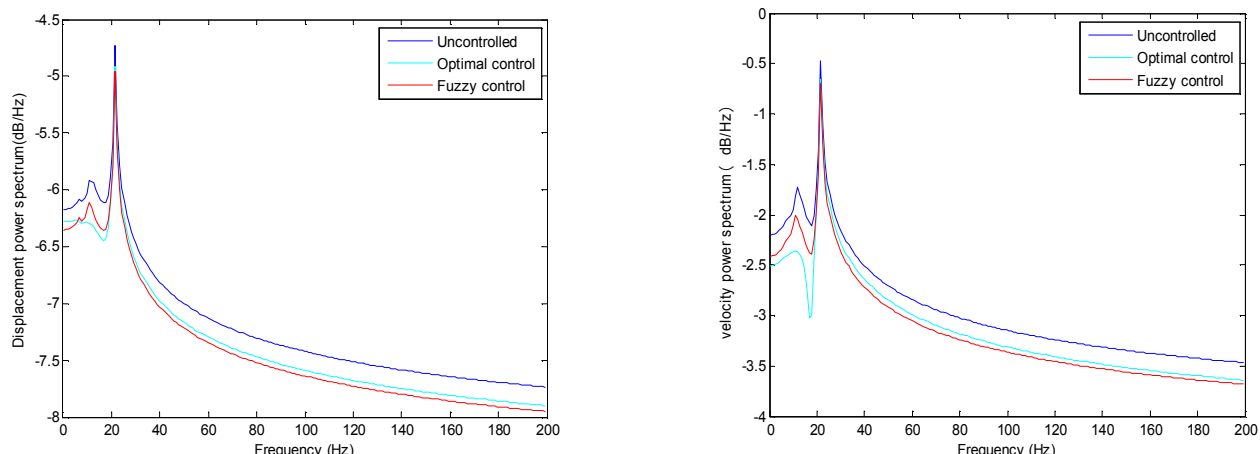

Fig. 5. Displacement and velocity frequency response when engine speed is $650 \mathrm{r} / \mathrm{min}$
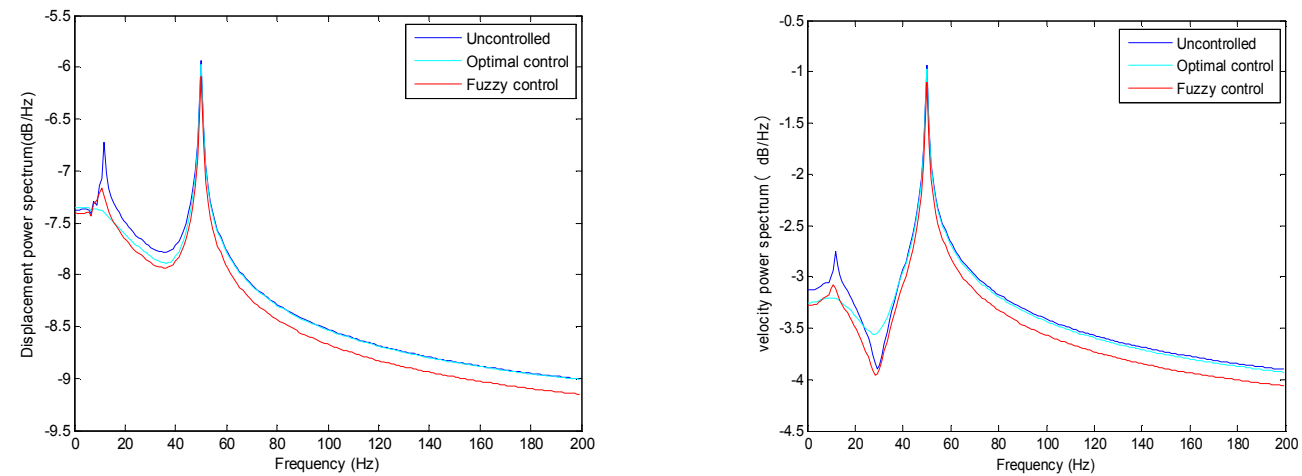

Fig. 6. Displacement and velocity frequency response when engine speed is $1500 \mathrm{r} / \mathrm{min}$

\section{Conclusion}

A mixed mode MR mount is designed to mitigate vibration and its vibration control performances are evaluated by considering the six degrees-of-freedom dynamic model. It has been demonstrated through the implementation of the semi-active optimal controller and fuzzy controller that the displacement and velocity at a certain MR mount can be both suppressed obviously.

\section{References}

[1] Kim G, Singh R. Nolinear analysis of automotive hydraulic engine mount. Journal of Dynamic System, Measurement and Control 1922;115(2): 482-7.

[2] Ushijima TK, Takano K, Kojima H. High performance hydraulic mount for improving vehicle noise and vibration. SAE Technical Paper Series 8800773.

[3] Elahinia M, Nguyan T, Ciocanel C, Parameter optimization in designing an MR mount, Proceeding of 15th International Congress on Sound and Vibration, 6-10 July, 2008, Daejeon, Korea. SS0561:237

[4] Young Kong Aha, Bo-Suk Yang, Mehdi Ahmadian, Shin Morishita, A Small-sized Variable-damping Mount using Magnetorheological Fluid, International Journal of Vehicle Design, Vol. 16 February 2005

[5] Sung-Ryong Hong, Seung-Bok Choi, Vibration Control of a Structural System Using Magneto-Rheological Fluid Mount, Journal International Material System And Structures, 2005

[6] Seung-Bok Choi, Sung-ryong Hong, Kum-Gil Sung, Jung-Woo Sohn, Optimal control of structural vibrations using a mixed-mode magnetorheological fluid mount. International Journal of Mechanical Sciences,2008, 50: 559-568 